\title{
Spatial Chaos in a Chain of Coupled Bistable Oscillators
}

\author{
V. I. Nekorkin and V. A. Makarov \\ Radiophysical Department, Nizhny Novgorod State University, 23 Gagarin Avenue, 603600 Nizhny Novgorod, Russia
} (Received 8 November 1994)

\begin{abstract}
The spatiotemporal behavior of a chain of diffusively coupled bistable oscillators is investigated. It is stated that there is spatial disorder and its evolutionary character is demonstrated.
\end{abstract}

PACS numbers: $05.45 .+b$

One of the problems of modern nonlinear physics attracting great attention of researchers is the investigation of chaotic behavior of dynamical systems. The phenomenon of finite-dimensional spatial disorder (spatial chaos) has been extensively studied [1-4]. Models in the form of nonlinear partial differential equations are usually used. Systems consisting of an infinite or a very great number of identical coupled maps, or ordinary differential equations, are also becoming more and more popular (see, e.g., [510]). These systems may be interpreted as coupled active elements located in the nodes of a discrete lattice in space, where the number of the element is a spatial coordinate. Such systems are called lattice dynamical models. Many systems in physics, chemistry, and biology can be described as populations of coupled self-excited oscillators. In particular, this sort of system has been considered in studies of biological oscillators [11], nonlinear synchronization networks [12], arrays of optical systems [13-15], Josephson arrays [16], etc.

Multistability (i.e., the existence of two or more stable states) is a fundamental property of nonlinear systems. It is clear that when such systems are combined into one common system the property of multistability influences essentially not only temporal but also the spatial behavior of this system. The general goal of this Letter is to study the spatiotemporal dynamics of a one-dimensional lattice model (i.e., a chain) written in the following form:

$$
\begin{gathered}
\ddot{x}_{j}+x_{j}=-\mu\left[f\left(x_{j}\right) \dot{x}_{j}-d\left(\dot{x}_{j-1}-2 \dot{x}_{j}+\dot{x}_{j+1}\right)\right], \\
j=1,2, \ldots, N, \quad \dot{x}_{0}=\dot{x}_{1}, \quad \dot{x}_{N+1}=\dot{x}_{N},
\end{gathered}
$$

where $0<\mu \ll 1$ and $f(x)$ are the parameter and the function characterizing dynamics of an individual unit of the chain, respectively, and $d$ is the coupling strength. Let us place on $f(x)$ the condition such that, for $d=0$, each unit of the chain (1) is a bistable oscillator, namely, $f(x)=a x^{4}-a x^{2}+1$, where $a>10$. In this case there is a fixed point at the coordinate origin and a stable limit cycle separated by an unstable limit cycle on the phase plane of an individual unit of the chain (1). Thus Eqs. (1) represent the chain of coupled bistable oscillators. The system (1) belongs to a class of basic models of nonlinear dynamics. Therefore such systems have been studied over and over again. The temporal dynamics (for example, phenomena of synchronization) has been studied in ample detail [17]. At the present time, many papers appear in which the primary attention is paid to spatial behavior of lattice models, for instance [8-10]. Among these works we note the interesting paper [8], where a nontrivial dependence of the wave-front velocity on $d$ was shown by numerical means. Here we will focus on the spatial disorder phenomenon in the chain (1).

System (1) is quasilinear and therefore it can be studied by averaging methods. An averaging system for (1) was obtained in [8] in the following form:

$$
\begin{gathered}
\dot{r}_{j}=-\mu F\left(r_{j}\right)+\mu d\left[r_{j-1} \cos \left(\varphi_{j-1}-\varphi_{j}\right)-2 r_{j}+r_{j+1} \cos \left(\varphi_{j+1}-\varphi_{j}\right)\right], \\
r_{j} \dot{\varphi}_{j}=\mu d\left[r_{j-1} \sin \left(\varphi_{j-1}-\varphi_{j}\right)+r_{j+1} \sin \left(\varphi_{j+1}-\varphi_{j}\right)\right], \\
j=1,2, \ldots, N, \quad r_{0}=r_{1}, \quad r_{N+1}=r_{N}, \quad \varphi_{0}=\varphi_{1}, \quad \varphi_{N+1}=\varphi_{N},
\end{gathered}
$$

where $r_{j}$ and $\varphi_{j}$ denote the amplitude and the phase of oscillations of the $j$ th unit, respectively, and $F(r)=$ $2 a r^{5}-a r^{3}+r$. It was shown in [8] that the system (2) is a gradient one and has a $2 \pi$-period Lyapunov function $U$. Thus, the evolution of any initial conditions is such that one of the equilibrium states of Eqs. (2) is realized. Let us now find the coordinates of these states.

To begin, let us note that only the states which satisfy $\varphi_{j-1}-\varphi_{j}=0$ may be stable. This conclusion follows from detailed analysis of the extremes of function $U$. It is obvious that the solutions $\varphi_{j}=\varphi^{0}$ satisfy this condition, where $\varphi^{0}$ is an arbitrary constant, i.e., the invariant line exists in the phase space of Eqs. (2). As far as the other coordinates of these equilibrium states are concerned, they are solutions of the equations

$$
\begin{gathered}
d\left(r_{j+1}-2 r_{j}+r_{j-1}\right)-F\left(r_{j}\right)=0, \\
r_{0}=r_{1}, \quad r_{N+1}=r_{N} .
\end{gathered}
$$

We set $u_{j}=r_{j-1}$, then (3) is rewritten as

$$
u_{j+1}=r_{j}, \quad r_{j+1}=2 r_{j}-u_{j}+d^{-1} F\left(r_{j}\right) \text {. }
$$


Equations (5) will be interpreted as a dynamical system on the plane determined by diffeomorfism $T$,

$$
(u, r) \rightarrow\left(r, 2 r-u+d^{-1} F(r)\right) .
$$

Then the portions of the discrete trajectories of this map $L:\left\{\left(u_{2}, r_{2}\right), \ldots,\left(u_{N}, r_{N}\right)\right\}$ on the $(u, r)$-phase plane joining the curves

$$
L_{2}:\left\{r=u+d^{-1} F(u)\right\}, \quad L_{N}:\left\{u=r+d^{-1} F(r)\right\},
$$

define the solutions of the system (3), (4) and, consequently, the equilibrium states of Eqs. (2). The map $T$ is a Henon-type map. The technique developed for the map of this type in [10] allows us to separate a domain in the parameter space, such that the map acts like the wellknown "Smale's horseshoe" map (see, e.g., [18]). Using this technique, we find the region $D$ determined by the following inequalities:

$$
\begin{gathered}
a>10, \\
d<\min \left\{\frac{a-10}{20}, \frac{F\left(r_{\max }\right)}{2\left(r_{3}^{0}-r_{\max }\right)}, \frac{-F\left(r_{\min }\right)}{2\left(r_{3}^{0}+r_{\min }\right)}\right\}, \\
\Phi\left(u_{\max }\right)>2 r_{3}^{0},
\end{gathered}
$$

where

$$
\begin{gathered}
r \min _{\max }=\sqrt{\frac{3 a \pm \sqrt{9 a^{2}-40 a}}{20 a}, \quad r_{3}^{0}=\sqrt{\frac{a+\sqrt{a^{2}-8 a}}{4 a}}}, \\
u \min _{\max }=\sqrt{\frac{3 a \pm \sqrt{9 a^{2}-40 a(1+2 d)}}{20 a}}, \\
\Phi(u)=2+d^{-1} F(u) .
\end{gathered}
$$

Note that $r_{\min }, r_{\max }$, and $r_{3}^{0}$ are the abscissas of the extremes and of the largest zero of the function $F(r)$, and $u_{\max }$ is the abscissa of the maximum of the function $\Phi(u)$. If the parameters of the map (5) belong to $D$, there is rectangle $\Pi$ on the $(u, r)$-phase plane, and $T$ acts like a Smale's horseshoe map on $\Pi$ (see Fig. 1). The sizes of $\Pi$ (quantities $\beta$ and $\gamma$ ) satisfy the inequalities

$r_{3}^{0}<\gamma<\beta, \quad \Phi(\gamma)-\gamma<\beta<\Phi\left(u_{\max }\right)-\gamma$,

and the images of the boundaries of the rectangle have the form (see Fig. 1)

$$
\begin{gathered}
T \Gamma^{ \pm}=\{(u, r)|u= \pm \gamma,| r-\Phi( \pm \gamma) \mid \leq \beta\}, \\
T B^{ \pm}=\{(u, r)|r=\Phi(u) \mp \beta,| u \mid \leq \gamma\} .
\end{gathered}
$$

Now we will be interested in the transformation of the initial curve $L_{2}$ by map $T$. Note that $r_{j} \geq 0$, and therefore we will consider the portions of $L_{2}$ which are located in the first quadrant of the plane $(u, r)$. For $0 \leq u \leq \gamma$, it follows from (7) that the curve $L_{2}$ is enclosed between $T B^{+}$and $T B^{-}$(Fig. 1). Consequently, the intersection of $L_{2}$ and rectangle $\Pi^{+}=\Pi \cap\{u \geq 0, r \geq 0\}$ has three components fitting into $\Omega_{i}^{+}=\Omega_{i} \cap\{u \geq 0, r \geq 0\}, \quad i=1,2,3$. These components are monotonically increasing curves joining the lines $r=0$ and $\Gamma^{+}$(see Fig. 1). We label the curves located inside $\Omega_{1}^{+}$and $\Omega_{3}^{+}$as $L_{2}^{0}$ and $L_{2}^{1}$, respectively.

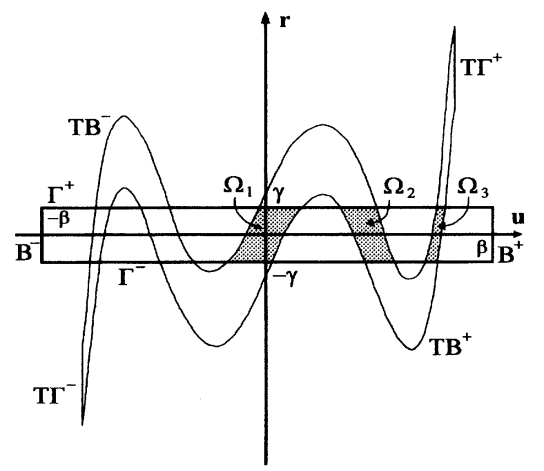

FIG. 1. The mapping of region $\Pi$ due to the iteration of $\operatorname{map} T$.

The solutions of Eqs. (3) and (4) corresponding to the trajectories of map $T$ passing through $\Omega_{2}^{+}$are unstable. The examination of the stability of solutions is carried out in the ordinary way using the Gerschgorin theorem [19]. According to the instability of above mentioned solutions, we omit from our consideration the portions of $T^{k} L_{2}$, $k=0,1,2, \ldots, N$, belonging to $\Omega_{2}^{+}$. As $L_{2}^{0}$ lies in $\Omega_{1}^{+}$, the iterations of $L_{2}^{0}$ occur by the "shoe" rules (recall that in $\Pi, T$ acts like a Smale's horseshoe map). Namely, $L_{2}^{0}$ is transformed to the curve $L_{3}$, which has one component in both $\Omega_{1}^{+}$and $\Omega_{3}^{+}$. We designate them as $L_{3}^{00}$ and $L_{3}^{01}$. These components are also monotonically increasing curves, similarly to $L_{2}$, and join the lines $r=0$ and $\Gamma^{+}$. Note that in the used notation $L_{k}^{0 m_{1} m_{2}, \ldots, m_{N-2}}$ the superscripts taking on the value 0 or 1 characterize the path traced by $L_{2}^{0}$ under iterations, and the subscript $k$ is associated with the number of iterations which is equal to $k-2$. Proceeding as before, we can divide the curve $L_{3}^{00}$ into two components $L_{4}^{000}$ and $L_{4}^{001}$, and $L_{3}^{01}$ into two components $L_{4}^{010}$ and $L_{4}^{011}$. Figure 2 illustrates the qualitative form of these components. So, the map $T$ splits each curve located in $\Omega_{1}^{+}$or $\Omega_{3}^{+}$into two. This process is shown schematically in Fig. 3. Thus after $N-2$ iterations, there appears a set consisting of $2^{N-2}$ monotonically increasing curves $L_{N}^{0 m_{1} m_{2}, \ldots, m_{N-2}}, m_{i} \in\{0,1\}$

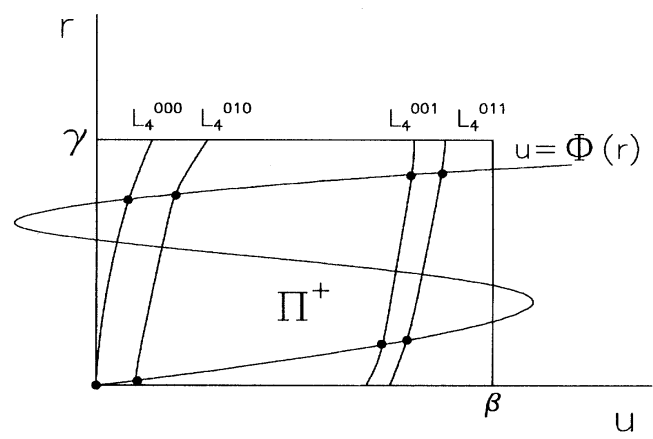

FIG. 2. The qualitative form of the components after the second division $L_{2}^{0}$. The dark dots mark the points of intersection components with $L_{N}$. 


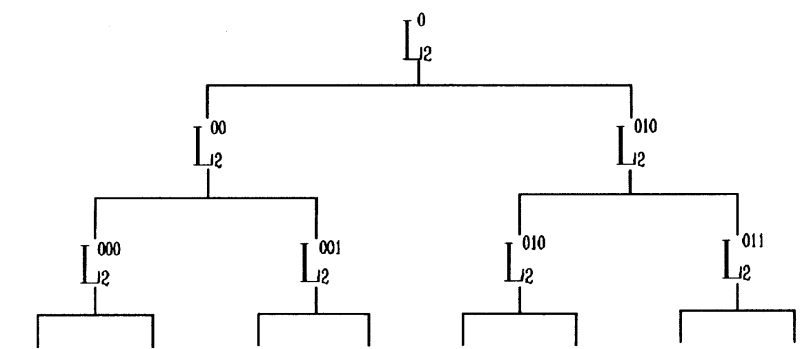

FIG. 3. Scheme of splitting the component $L_{2}^{0}$ by map $T$.

joining the lines $r=0$ and $\Gamma^{+}$and located in the domains $\Omega_{1}^{+}$and $\Omega_{3}^{+}$. A similar process takes place for the transformation component $L_{2}^{1}$. It results in a set consisting of $2^{N-2}$ curves $L_{N}^{1 m_{1} m_{2}, \ldots, m_{N-2}}, m_{i} \in\{0,1\}$ joining the lines $r=0$ and $\Gamma^{+}$. Thus, on the one hand, the set consisting of $2^{N-1}$ curves $L_{N}^{0 m_{1} m_{2}, \ldots, m_{N-2}}$ and $L_{N}^{1 m_{1} m_{2}, \ldots, m_{N-2}}$ is formed as a result of $N-2$ iterations of $L_{2}^{0}$ and $L_{2}^{1}$. On the other hand, only the portions of the trajectories of map $T$ which starts on $L_{2}^{0}$ or $L_{2}^{1}$ and get to $L_{N}$ are of interest to us. It is clear that inside the rectangle $\Pi^{+}$ $L_{N}$ has two monotonically increasing curves joining the lines $u=0$ and $B^{+}$. That is why $L_{N}$ intersects both $L_{N}^{0 m_{1} m_{2}, \ldots, m_{N-2}}$ and $L_{N}^{1 m_{1} m_{2}, \ldots, m_{N-2}}$ at two points. As a result of these intersections there exist $2^{N}$ points located in the increasing portions of the curve $L_{N}$ [the points lying in the decreasing portion of $L_{N}$ are not considered because they correspond to the maxima of the function $U$ and unstable solutions of Eqs. (2)]. Hence, $2^{N}$ portions of trajectories of map $T$ joining $L_{2}$ and $L_{N}$ exist in the first quadrant of the $(u, r)$-phase plane. The coordinates of the points of these portions determine $2^{N}$ sets of solutions $r_{j}=r_{j}^{*}, j=1,2, \ldots, N$, of the system (3) and (4). Note that there are two homogeneous solutions independent of $j:\left\{r_{j}=0\right\}$ and $\left\{r_{j}=r^{*}\right\}$.

Thus the system (2) has $2^{N}$ equilibrium states with the coordinates $r_{j}=r_{j}^{*}, \varphi_{j}=\varphi^{0}$, where $j=1,2, \ldots, N$. Consequently, the oscillations in the chain (1) are synchronized. At this regime the distribution of oscillation amplitudes along the chain is described by the map (5) and can be very complex.

In order to verify and supplement some of the theoretical predictions made above we simulated Eqs. (1) numerically. First, we considered a synchronization of oscillators in the chain. To this end, we integrated the system of ten oscillators. (All numerical results were obtained with $a=14, d=0.07$, and $\mu=0.01$.) The main results of the experiments may be summarized as follows. The process of the onset of synchronous motions may be divided into two stages having different time scales. (i) Formation of clusters. (Here we define a cluster as a set of oscillators in which $\varphi_{i} \cong \varphi_{j}$ for $i, j$ belonging to one set.) At this stage, the oscillators are divided into some groups in which the oscillators are phase locked. Figure 4(a) illus- trates the process of formation of two clusters. (ii) Interaction of clusters. At this stage, the clusters interact with one another, as a result of which the phase difference between clusters changes. However, this difference does not become equal to zero as it follows from the investigation of Eqs. (2). It is explained by the fact that we observe a quasiperiodic solution of Eqs. (1) in numerical experiment $\left(\varphi^{0}\right.$ is the first component of asymptotic expansion of a phase of this solution). In the interaction process, clusters move very slowly. The distribution of oscillator amplitudes essentially influences this process. Figure 4(b) shows the phase distribution at different instants of time.

We now consider the amplitude distribution. We integrated Eqs. (1) where $N=40$. The initial amplitude
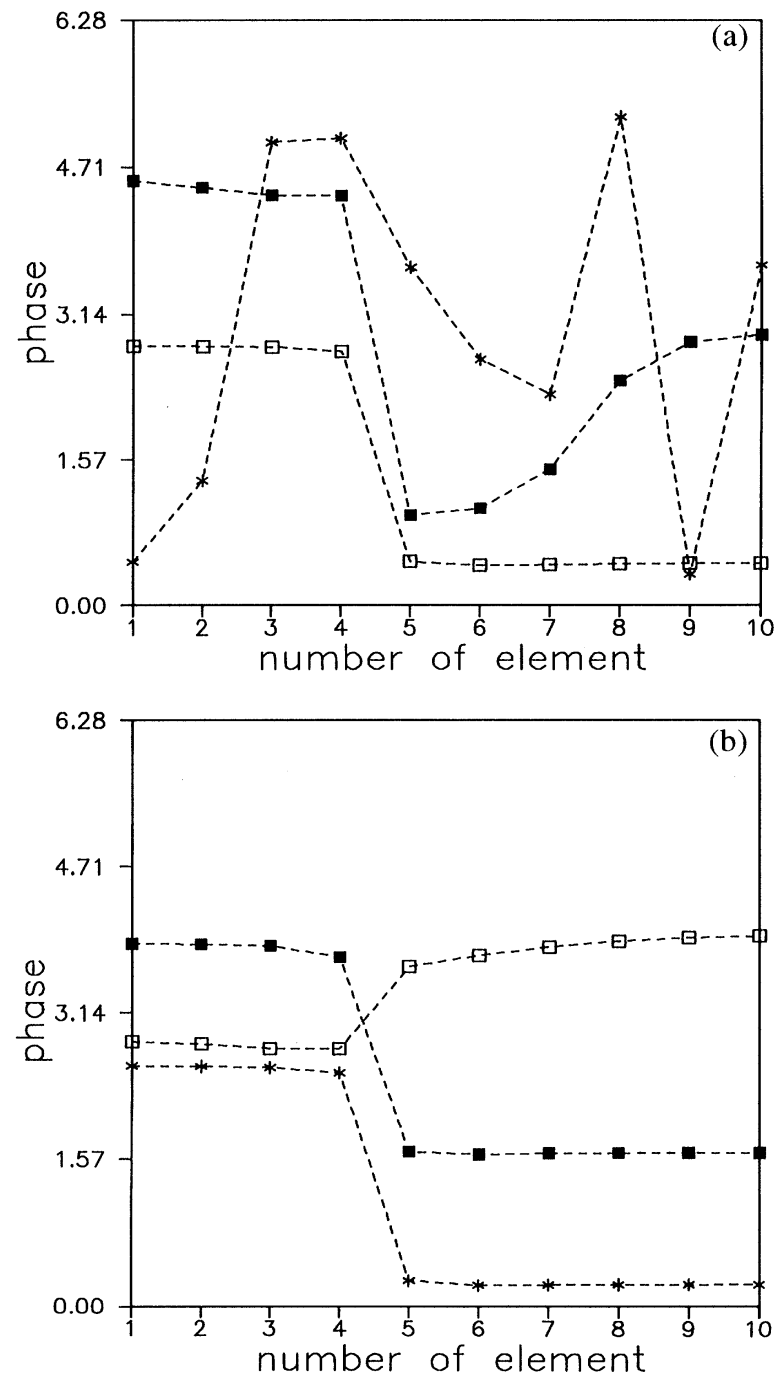

FIG. 4. The mean phase vs the number of oscillator at three different instants of time: (a) $t=0$ (asterisks), $t=8000$ (dark squares), and $t=45000$ (squares); (b) $t=90000$ (asterisks), $t=220000$ (dark squares), and $t=300000$ (squares). 


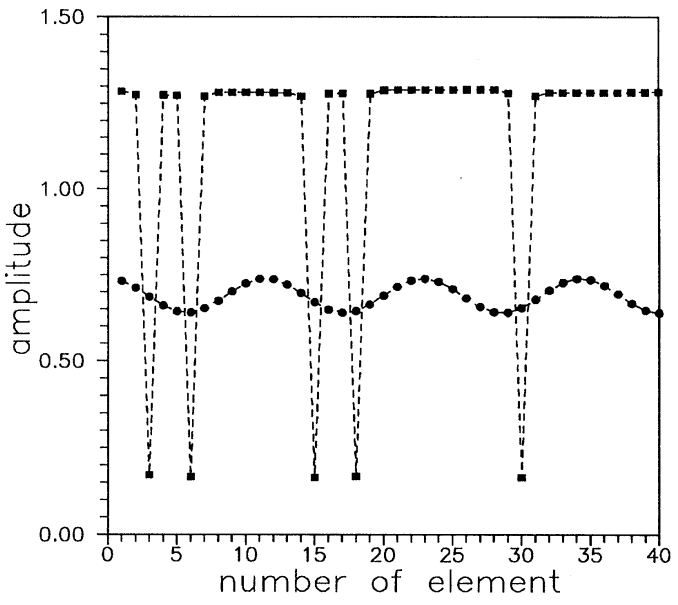

FIG. 5. The dependence of amplitude of oscillations on the number of oscillator (circles, initial; squares, final).

distributions were chosen to be sinusoidal. Our experiments show that the system has high sensitivity to initial distributions of $x(t)$. Note that the sensitivity is peculiar to a large class of spatially extended systems [3]. Figure 5 illustrates a typical initial and final distribution of amplitude of oscillations. In the course of amplitude distribution, phase singularities are formed at the values of $j$ corresponding to inhomogeneous amplitude distribution.

In conclusion, we summarize the results of our work. The chain (1) represents a nonequilibrium medium, i.e., the processes are developing both in time and in space. In time at the units of the chain occur oscillations with the same frequency. The process of synchronization takes place through clustering. The amplitude distribution of oscillations along the spatial coordinate $j$ is determined by the portions of the trajectories of map $T$ and can be described by a sequence of two symbols $\left(0, m_{2}, m_{3}, \ldots, m_{N-1}\right),\left(1, m_{2}, m_{3}, \ldots, m_{N-1}\right)$, where $m_{i} \in$ $\{0,1\}$. Moreover, the symbol " 0 " corresponds to a small enough amplitude of oscillations, and the symbol " 1 " corresponds to the amplitude that is close to the amplitude of a limit cycle of an individual oscillator that is not affected by the neighboring oscillators. There are $2^{N}-1$ such motions, hence the system (1) is a multistable one. Since the map $T$ has chaotic dynamics, the alternations of the symbols " 0 " and "1" will be quite diverse and, for
$N \rightarrow \infty$, can be described by the Bernoulli shift. Consequently, the oscillations in the chain (1) are regular in time and disordered in space. The finite spatial disorder in the chain is evolutionary (i.e., it can be formed out of order) as the averaging system (2) is a gradient one.

We thank M.I. Rabinovich and M.G. Velarde for useful discussions.

[1] M. I. Rabinovich, A. L. Fabrikant, and L. Sh. Tsimring, Sov. Phys. Usp. 35, 629 (1992).

[2] P. Collet and J-P. Eckmann, Nonlinearity 5, 126 (1992).

[3] C. Nicolis, G. Nicolis, and Q. Wang, Int. J. Bifurcation Chaos 2, 263 (1992).

[4] P. Coullet, C. Elphick, and D. Repaux, Phys. Rev. Lett. 58, 431 (1987).

[5] I. S. Aranson, A. V. Gaponov-Grekhov, M. I. Rabinovich, A. V. Rogal'skii, and R. V. Sagdeev (to be published).

[6] L. A. Bunimovich and Ya. G. Sinai, Nonlinearity 1, 581 (1988).

[7] K. Kaneko, Physica (Amsterdam) 37D, 60 (1989).

[8] A-D. Defontaines, Y. Pomeau, and B. Rostand, Physica (Amsterdam) 46D, 201 (1990).

[9] A. Greenfield, S. Putterman, and W. Wright, Phys. Lett. A 185, 321 (1994).

[10] V.S. Afraimovich and V.I. Nekorkin, Int. J. Bifurcation Chaos 4, 631 (1994).

[11] J.D. Murray, Mathematical Biology (Springer-Verlag, New York, 1991).

[12] V.S. Afraimovich, V. I. Nekorkin, G. V. Osipov, and V. D. Shalfeev, Stability, Structures, and Chaos in Nonlinear Synchronization Networks (World Scientific, Singapore, 1995).

[13] R. Li and T. Erneux, Phys. Rev. A 49, 1301 (1994).

[14] W. Rappel, Phys. Rev. E 49, 2750 (1994).

[15] G. Dangelmayr and M. Kirby, Int. Series Numerical Math. 104, 85 (1992).

[16] S. Nichols and K. Wiesenfeld, Phys. Rev. E 50, 205 (1994).

[17] Ju. M. Romanovsky, N. V. Stepanova, and D.S. Chernavsky, Mathematical Simulation in Biophysics (Nauka, Moscov, 1975).

[18] Z. Nitecki, Differentiable Dynamics: An Introduction to the Orbit Structure of Diffeomorphisms (MIT, Cambridge, 1971).

[19] R. A. Horn and C. R. Johnson, Matrix Analysis (Cambridge University, Cambridge, 1986). 